\title{
Gyrokinetic and gyrofluid investigation of magnetic islands in tokamaks
}

\author{
E. Poli, A. Bottino, W. A. Hornsby ${ }^{1}$, A. G. Peeters ${ }^{1,2}$, T. Ribeiro, B. D. Scott, M. Siccinio \\ Max-Planck-Institut für Plasmaphysik, Garching bei München, Germany \\ 1 Centre for Space and Astrophysics, \\ Department of Physics, University of Warwick, UK and \\ ${ }^{2}$ Department of Physics, University of Bayreuth, Germany
}

\begin{abstract}
The evolution of a tearing mode is a multi-scale problem, involving lengths from below the ion gyroradius up to the dimensions of the system. The effects due to finite ion Larmor radius on the island dynamics are investigated by means of numerical gyrokinetic and gyrofluid simulations in tokamak geometry. In gyrokinetic runs, the magnetic island is prescribed. The coupling induced by a static island between small and large scale fluctuations in the case of electrostatic turbulence is discussed and the role of the perturbed magnetic geometry on the electron response is highlighted. Simulations in the presence of a rotating island, excluding background turbulence, allow a clear, self-consistent determination of the electrostatic potential associated to the island rotation and of the relevant plasma profiles for arbitrary island widths. Finally, the first gyrofluid simulations showing the growth of an island in the presence of electromagnetic turbulence for parameters typical of a mid-size tokamak are presented.
\end{abstract}




\section{INTRODUCTION}

The tearing instability[1, 2], appearing as magnetic islands developing on low-order rational surfaces, is often observed in tokamak experiments. A fully-developed tearing mode represents a serious concern for a tokamak reactor, as it limits the achievable plasma pressure and can trigger disruption events[3]. In axisymmetric devices, the dynamics of the tearing mode is strongly affected by toroidal effects. The mode is often neoclassically driven by the drop of the bootstrap current caused by pressure flattening within the reconnected region[46]. Moreover, the neoclassical polarization current[7, 8] and Pfirsch-Schlüter currents[9-11] have been demonstrated to influence the island evolution. More recently, the interaction between small-scale turbulence and "mesoscale" magnetic islands has attracted a growing attention, also because of the fact that the increasing availability of high-performance numerical resources allows the exploration of more and more realistic parameters. The importance of this mutual influence between different scales for magnetic reconnection has been long recognized in general[12] and for magnetic islands in fusion plasmas in particular[13]. As far as the influence of small on large scales is concerned, besides the investigation of turbulence as a source of anomalous dissipation (viscosity, resistivity)[14-18], electromagnetic turbulence has been shown to be able to provide a trigger for the growth of an island[19]. Moreover, turbulent transport contributes to determining the density and temperature profiles across the reconnected region, in turn influencing the contribution of bootstrap[20] and polarization[21] current to the island dynamics. On the other side, the tearing mode has a major impact on the turbulence profile, as the above-mentioned pressure flattening inside the island reduces the drive for the instabilities. In addition, the turbulence is regulated by the sheared flows associated with the long-wavelength modes (this last effect being similar to that of zonal flows in axisymmetric systems). A nice overview of the properties of the mutual interaction between small and large-scale instabilities has been given by McDevitt and Diamond[17].

A magnetic island is a system exhibiting different spatial scales along and across it. In tokamaks, the poloidal wavelength of the island is of the order of the minor radius, while its width can be of the order of the ion Larmor radius or below. For this reason, kinetic studies of the tearing-mode dynamics employing the gyrokinetic theory have been already carried on in the past. Slab-geometry calculations have been performed to investigate analytically 
the problem of the island propagation and the role of the polarization current, the emission of drift waves, and their stability in the presence of an island-perturbed equilibrium[22-25]. Numerical gyrokinetic particle-in-cell (PIC) simulations have been employed to study the reconnection problem in a collisionless plasma slab[26] and also extended to the semicollisional regime[27]. On the other hand, the gyroradius is the typical spatial scale of turbulent fluctuations in fusion plasmas. For a description of the effects of the presence of a tearing mode on microturbulence and vice versa, finite-Larmor-radius (FLR) effects must then be retained, by means of a gyrofluid or gyrokinetic approach.

It is clear that the self-consistent evolution of island and turbulence constitutes a formidable multi-scale problem. On the way to its complete solution, several routes are being explored, that reduce the size of the problem by means of appropriate simplifications. This paper is intended to present recent new results on the role of FLR effects on the dynamics of magnetic islands in toroidal plasmas. We adopt two different numerical approaches, the first based on gyrokinetic, the second on gyrofluid equations. In the former case, we rely on the separation between the typical time scales involving the development turbulence and the island growth (for a discussion see also Refs. 21, 28). In this scheme, the magnetic island is prescribed, i. e. island rotation and width do not evolve. This excludes, of course, the feedback of short on long time scales. On the other side, toroidicity and kinetic effects are retained. They are known to significantly change the bootstrap[29-31] and polarization current[32-34] and are, hence, potentially important for the "closure" of the interaction loop, when neoclassical effects on the mode evolution will be considered. Two sets of gyrokinetic simulations are presented here. First, we explore the behaviour of electrostatic turbulent fluctuations in the presence of a static island, that confirm and extend, in particular through the inclusion of the perturbed magnetic geometry in the electron response[35], previous recent investigations[36]. The second part of our gyrokinetic results refers to a rotating island, for which the self-consistent electrostatic potential and the pressure profile are investigated switching off the turbulence. These simulations, that represent a first steps towards a neoclassical analysis, are found to support previous drift kinetic results[31]. The latter (gyrofluid) approach mentioned before implies a different simplification of the problem (namely, neglecting kinetic effects) but allows a simultaneous treatment of turbulence and island time-scales. Here, the first results obtained for a set of plasma parameters close to those typical of the ASDEX Upgrade tokamak are presented. It is worth noting that a fluid 
approach has been already employed recently to resolve both turbulence and island scales in both slab geometry[37] and including curvature effects[38, 39].

In the next section, the numerical tools employed in our simulations, namely the two gyrokinetic codes ORB5[40, 41] (particle-in-cell, global) and GKW[42, 43] (Vlasov, fluxtube) and the global gyrofluid code GEMZ[44] are briefly presented, with emphasis on the different treatment of the magnetic perturbation associated to the magnetic island. Gyrokinetic results in the presence of turbulence are discussed in Section III, while the simulations of a rotating island in the absence of turbulence are presented in Section IV. Section $\mathrm{V}$ is finally devoted to gyrofluid simulations. A summary and discussion of the results can be found in Section VI.

\section{NUMERICAL TOOLS}

The dynamics of microinstabilities in toroidal geometry in the presence of a magnetic island is investigated in this paper employing the two complementary schemes described above, namely a gyrokinetic and a gyrofluid approach. A magnetic island developing on a given rational surface $\psi_{s}$ characterized by a poloidal number $m$ and toroidal number $n$ can be introduced as a perturbation of the parallel vector potential (or, equivalently, of the poloidal flux $\tilde{\psi}=-R \tilde{A}_{\|}, R$ being the major radius of the tokamak) of the form $\tilde{A}_{\|}=\bar{A}_{\|} \cos \xi$, where $\xi=m \theta-n \varphi-\omega t$ is the helical angle ( $\theta$ and $\varphi$ being the poloidal and toroidal angle, respectively, and $\omega$ the island rotation frequency). The corresponding perturbed magnetic field is mainly directed radially and varies in the $\nabla \xi$ direction as

$\sin \xi$. A new flux label $\Omega=2\left(\psi-\psi_{s}\right)^{2} / W_{\psi}^{2}-\cos \xi$, where $W_{\psi}=\sqrt{4 R \bar{A}_{\|} q_{s} / q_{s}^{\prime}}$ is the island width in $\psi$ units (the prime denoting differentiation with respect to $\psi$ ), can be introduced such that the total magnetic field is perpendicular to $\nabla \Omega$. With this definition, $\Omega$ varies between -1 at the island O-point (where $\psi=\psi_{s}$ and $\xi=0$ ) and $+\infty$. The island width $W$ is obtained in terms of $W_{\psi}$ as $W=W_{\psi} /|\nabla \psi|$.

\section{A. Gyrokinetic approach}

Two gyrokinetic codes have been used in our study, based on different methods for the solution of the gyrokinetic equation. 
The global PIC code ORB5 provides a numerical solution to the gyrokinetic equations in the formulation of Hahm[45]. It employs the particle-in-cell method, which is based on the introduction of an ensemble of numerical particles (markers), each one connected with a piece of the phase space associated with a given particle species. In the simulations presented here, the electrons are adiabatic and the markers represent only the main ion species $i$. The evolution of the markers is determined by the corresponding equations of motion, which are coupled to Maxwell's equations. The self-consistent fields are calculated projecting the charge and current associated with each marker onto a fixed spatial grid (in this paper, only simulations of electrostatic turbulence will be considered). The total distribution function is split into an analytically-known time-independent part $f_{0}$ and a perturbation $\delta f$ which is represented numerically. The gyrokinetic equations of motion for the markers are

$$
\begin{aligned}
\frac{\mathrm{d} \mathbf{R}}{\mathrm{d} t} & =v_{\|} \mathbf{b}+\frac{1}{B_{\|}^{*}}\left[\frac{\mu B+v_{\|}^{2}}{\Omega_{c}} \mathbf{b} \times \nabla B-\frac{v_{\|}^{2}}{\Omega_{c}} \mathbf{b} \times(\mathbf{b} \times \nabla \times \mathbf{B})-\nabla\langle\tilde{\phi}\rangle_{g} \times \mathbf{b}\right], \\
\frac{\mathrm{d} v_{\|}}{\mathrm{d} t} & =-\mu\left[\mathbf{b}-\frac{v_{\|}}{B_{\|}^{*} \Omega_{c}} \mathbf{b} \times(\mathbf{b} \times \nabla \times \mathbf{B})\right] \cdot \nabla B \\
& -\frac{Z e}{M}\left\{\mathbf{b}+\frac{v_{\|}}{B_{\|}^{*} \Omega_{c}}[\mathbf{b} \times \nabla B-\mathbf{b} \times(\mathbf{b} \times \nabla \times \mathbf{B})]\right\} \cdot \nabla\langle\tilde{\phi}\rangle_{g}, \\
\frac{\mathrm{d} \mu}{\mathrm{d} t} & =0,
\end{aligned}
$$

where $\mathbf{R}$ is the position of the gyrocentre, $v_{\|}$the velocity component along the magnetic field, $\mathbf{b}$ the unit vector along the magnetic field $\mathbf{B}, \mu$ the magnetic moment, $\Omega_{c}$ the cyclotron frequency, $\langle\tilde{\phi}\rangle_{g}$ the perturbed potential (solution of the Poisson equation) averaged over the gyroperiod, $Z e$ and $M$ the particle's charge and mass, respectively, and $B_{\|}^{*}=B+(M / Z e) v_{\|} \mathbf{b} \cdot \nabla \times \mathbf{b}$. Since along the orbits $\mathrm{d} f / \mathrm{d} t=0$ (no collisions are considered here), $\delta f$ must obey the equation

$$
\frac{\mathrm{d}(\delta f)}{\mathrm{d} t}=-\frac{\mathrm{d} f_{0}}{\mathrm{~d} t}=-\frac{\mathrm{d} \mathbf{R}}{\mathrm{d} t} \cdot \nabla f_{0}-\frac{\mathrm{d} v_{\|}}{\mathrm{d} t} \frac{\partial f_{0}}{\partial v_{\|}} .
$$

The velocity $\mathrm{d} \mathbf{R} / \mathrm{d} t$ includes the radial motion along the perturbed field lines. The radial component $\tilde{\mathbf{B}}$ of the magnetic field produced by the tearing mode accounts for the most important modification of particle orbits[46] and has been included in ORB5 by operating the substitution $\mathbf{b} \rightarrow \mathbf{b}+\tilde{\mathbf{b}}$ (where $\tilde{\mathbf{b}}=\tilde{\mathbf{B}} / B$ ) in the first term of both Eq. (1) and Eq. (2). 
In the Vlasov flux-tube code GKW, the gyrokinetic equation is solved in the form

$$
\frac{\partial g}{\partial t}+\mathbf{v}_{\chi} \cdot \nabla g+\left(v_{\|} \mathbf{b}+\mathbf{v}_{d}\right) \cdot \nabla(\delta f)-\frac{\mu B}{M} \frac{\mathbf{B} \cdot \nabla B}{B^{2}} \frac{\partial(\delta f)}{\partial v_{\|}}=S
$$

on a five-dimensional grid in the $\left(\mathbf{X}, \mu, v_{\|}\right)$space, where $\mathbf{X}$ is the gyro-centre position (the index denoting the particle species is suppressed for brevity). In the previous equation, the distribution function is split into a Maxwellian background term $F_{M}$ and a deviation $\delta f$, with the auxiliary function $g$ defined as $g=\delta f+(Z e / T) v_{\|}\left\langle\tilde{A}_{\|}\right\rangle_{g} F_{M}$. The velocity $\mathbf{v}_{\chi}=(\mathbf{b} \times \nabla \chi) / B$, with $\chi=\langle\tilde{\phi}\rangle_{g}+v_{\|}\left\langle\tilde{A}_{\|}\right\rangle_{g}$, is a combination of the $\mathbf{E} \times \mathbf{B}$ velocity and the parallel motion along the perturbed field lines, and $\mathbf{v}_{d}$ is the drift velocity due to magnetic-field inhomogeneity ( $\nabla B$ and curvature). The electrostatic potential is the solution of the Poisson equation, while $\tilde{A}_{\|}$is the imposed island perturbation. The source term on the right-hand side of Eq. (5) reads as

$$
S=-\left(\mathbf{v}_{\chi}+\mathbf{v}_{d}\right) \cdot\left[\frac{\nabla n}{n}+\left(\frac{v_{\|}^{2}}{v_{\mathrm{th}}^{2}}+\frac{\mu B}{T}-\frac{3}{2}\right) \frac{\nabla T}{T}\right] F_{M}-\frac{Z e}{T}\left(v_{\|} \mathbf{b}+\mathbf{v}_{d}\right) \cdot \nabla\langle\tilde{\phi}\rangle_{g} F_{M}
$$

( $n$ and $T$ are the background density and temperature, respectively, and $v_{\text {th }}=\sqrt{2 T / M}$ is the thermal velocity). In the previous equations, the contribution connected to the radial profile of the toroidal plasma rotation velocity has been omitted. With respect to the global approach described above, the physical model remains the same, but the equations are solved in a finite domain of the plasma around a given field line. Correspondingly, the socalled flux-tube ordering[47] is adopted, in which the deviation of the distribution function from its equilibrium value is assumed to be small over the region of interest, whereas its gradients can be comparable to the equilibrium gradients. For a more detailed discussion of the ordering assumptions in GKW see Refs. 43, 48 (a comparison between the global and flux-tube results is presented in Section III). GKW is written in field-aligned Hamada coordinates $(\psi, \zeta, s)$. When a magnetic equilibrium with circular concentric flux surfaces is adopted, $s$ can be regarded as a normalized poloidal angle varying between $-1 / 2$ and $1 / 2(s=\theta / 2 \pi$ in the large-aspect-ratio limit $\epsilon \rightarrow 0)$ and $\zeta=(q \theta-\varphi) / 2 \pi$ is chosen such that the parallel-gradient operator $\mathbf{B} \cdot \nabla$ involves only $\partial / \partial s$. In these coordinates, the helical angle $\xi$ can be expressed in the vicinity of the rational surface, which is supposed to be located at the centre of the computational box $\left(\bar{\psi}=\psi-\psi_{s}\right.$ being the distance from this surface), expanding the safety factor appearing in the definition of $\zeta$ to the first order 
around $q_{s}=m / n$,

$$
m \theta-n \varphi-\omega t=2 \pi\left(n \zeta-n s q_{s}^{\prime} \bar{\psi}\right)-\omega t=k_{\zeta} \zeta-s k_{\psi} \bar{\psi}-\omega t
$$

Here, the island wave vector with components $k_{\zeta}=2 \pi n \rho_{*}$ and $k_{\psi}=q_{s}^{\prime} k_{\zeta}$ has been introduced (the wave vector is expressed in units of $1 / \rho_{i}$, with the definition $\rho_{*}=\rho_{i} / R$, $\rho_{i}$ being the ion gyroradius). This expression shows that the periodicity constraint in the radial direction (required by the spectral approach adopted in GKW) cannot be satisfied for every value of $s$. Defining the radial width of the box as $\Delta \psi=\left[-\pi / k_{\psi}, \pi / k_{\psi}\right]$, the first step to impose periodicity is projecting the vector potential on the $\psi$-harmonics,

$$
\tilde{A}_{\|}=e^{i\left(k_{\zeta} \zeta-\omega t\right)} \sum_{p=0}^{\infty} A^{p}(s) e^{i p k_{\psi} \bar{\psi}}
$$

with

$$
A^{p}(s)=\frac{k_{\psi}}{2 \pi} \bar{A}_{\|} \int_{-\pi / k_{\psi}}^{\pi / k_{\psi}} e^{-i p k_{\psi} \bar{\psi}} e^{-i s k_{\psi} \bar{\psi}} \mathrm{d} \psi=\bar{A}_{\|} \frac{\sin [\pi(s+p)]}{\pi(s+p)} .
$$

This simple implementation turns out to be unsatisfactory, as it leads to abrupt jumps in the vector potential at the edge of the radial computational domain, where periodicity is enforced (Fig. 1a). The problem, however, can be circumvented by introducing a smoothing factor at high $p$-harmonics (Fig. 1b), i. e. by operating the replacement

$$
\frac{\sin [\pi(s+p)]}{\pi(s+p)} \rightarrow \exp \left[-(s+p)^{2} / L^{2}\right] \frac{\sin [\pi(s+p)]}{\pi(s+p)}
$$

in the previous equation. The exact value of the scale $L$ does not affect the results, provided it is large enough.

\section{B. Gyrofluid approach}

Following the dynamics of magnetic islands and microturbulence simultaneously is not presently feasible by gyrokinetic models without unphysical compromises involving the parameters of scale separation. If it is to be demonstrated that small and large scales can interact, then the results are only meaningful with the correct values of the dimensionless ratios $\rho_{s} / a$ or $M_{e} / M_{i}$ or $\beta_{e}=c_{s}^{2} / v_{A}^{2}$ or $q R / L_{T}$, where $c_{s}$ is the sound speed, $v_{A}$ is the Alfvén speed, and $\rho_{s}$ is the drift scale (also called the ion sound gyroradius). See Refs. 49, 50 for the significance these parameters, alone and in combination, have for tokamak microturbulence. A moderate sized tokamak has $a / \rho_{s} \sim 200$, a deuterium plasma 
has $M_{i} / M_{e}=3670$, and typical conditions have $\beta_{e} \sim 3 \times 10^{-3}$ for the plasma core. The electromagnetic gyrofluid equations are as given in Ref. 44. Time dependent equations for six gyrofluid moment variables (for each species, density, parallel velocity, parallel and perpendicular temperature and parallel and perpendicular heat fluxes associated to each temperature) are advanced for a singly charged, single component, purely ionized plasma. The self consistent fields $\tilde{\phi}$ and $\widetilde{A}_{\|}$are found via the polarization and induction equations, respectively. The tilde symbol denotes a dependent variable. The corresponding free energy conservation law, also given there, is relevant because the energetic contact between the electromagnetic microturbulence and the much slower large-scale MHD through much faster shear-Alfvén transients involves a small amount of energy which however yields strong consequences. The only modification in the equations as used here is the incorporation of the current profile as a free energy source, as described in Section V. The flux surface geometry is built around the equilibrium flux surfaces. Islands are initialized or emerge naturally and are represented as part of the $\tilde{A}_{\|}$disturbances.

For this work the geometry is updated by using field-aligned conformal coordinates. Fieldaligning means using a Clebsch representation $\{x y s\}$, in which $x(\psi)$ is a surface label of the poloidal flux, $s$ is a parallel coordinate set equal to either the poloidal or toroidal angles, and $y$ is a function of both angles but through the use of the pitch parameter $q(\psi)$ satisfies $B^{y} \equiv \mathbf{B} \cdot \nabla y=0$ exactly, everywhere. This is only possible for straight field line angle coordinates because $q=B^{\varphi} / B^{\theta}$ and to build Clebsch coordinates $\mathbf{B}=\chi(x) \nabla x \times \nabla y$ the condition $q=q(\psi)$ is necessary[51]. The function $\chi$ gives effective normalization. Here, $\varphi$ is the geometric toroidal angle and the choice $s=\varphi$ leads to $y_{k}=\theta-q^{-1}\left(\varphi-\varphi_{k}\right)$ with the constant $\varphi_{k}$ giving the reference position. The use of a different $\varphi_{k}$ on each poloidal plane $\varphi=\varphi_{k}$ is a version of the shifted metric procedure[52] which avoids secular deformation in the metric coefficient ratio $g^{x y} / g^{x x}$. Here, the sign convention is for $\mathbf{B}=I \nabla \varphi+\nabla \varphi \times \nabla \psi$ and $\nabla R \times \nabla Z \cdot \nabla \varphi>0$.

The linear component of the parallel derivative is calculated as $\mathbf{B} \cdot \nabla=B^{s}(\partial / \partial s)$. The conformal property is then introduced to further avoid poloidally periodic deformation in the coordinate cells, by stating $\mathbf{B} \cdot \nabla \theta_{c}=\left(f_{c} / R\right)|\nabla \psi|^{2}$ as the poloidal angle definition, where $f_{c}(\psi)$ is the conformal flux function found by normalizing $\theta_{c}$ to a $2 \pi$-cycle for periodicity. The radial coordinate $x_{c}(\psi)$ is defined as $\nabla x_{c}=f_{c} \nabla \psi$. The straight field line angle definition, by contrast, is defined as $\mathrm{B} \cdot \nabla \theta=I / q R^{2}$ since $B^{\varphi}=I / R^{2}$, with $q$ found by 
normalizing $\theta$ to a $2 \pi$-cycle. We define $y_{c}=\theta_{c}$. The relationships $\theta_{c}(\theta)$ and $\theta\left(\theta_{c}\right)$ are used to interpolate the finite difference of $\partial / \partial s$ onto a grid which is equidistant in $x_{c}$ and $y_{c}$ in the poloidal plane. This makes use of the fact that $y=\theta$ for $\varphi=\varphi_{k}$. One can then derive the coordinate Jacobian $\nabla x_{c} \times \nabla y_{c} \cdot \nabla s=g_{c}^{x x} / R$ and the deformation $d=g_{c}^{x y} / g_{c}^{x x}$ using the definitions of $f_{c}$ and $x_{c}$. One finds that in conventional tokamak equilibrium models $d^{2}<1$ almost everywhere, facilitating both the representation of microturbulence and iterative solvers for linear operators involving the perpendicular Laplacian $\nabla_{\perp}^{2}$, and that the conformal property $g_{c}^{x x} g_{c y y}=1$ is exactly satisfied. In the cylindrical limit one can show $y_{c}$ and $\theta$ both relax to $\eta$, the geometric axial angle, and $x_{c}$ relaxes to $\log r$ with $r$ the cylindrical radius. The subscript $c$ in each of these expressions denotes the conformal coordinate system $\left\{x_{c}, y_{c}, \varphi\right\}$. For further motivation and details see Ref. 51 .

\section{GYROKINETIC SIMULATIONS OF ITG TURBULENCE IN THE PRES- ENCE OF A MAGNETIC ISLAND}

Vlasov flux tube and global PIC simulations are employed to investigate the behaviour of electrostatic ion-temperature-gradient (ITG) driven turbulence in the presence of a prescribed $(m=3, n=2)$ magnetic island. The parameters employed in the runs of GKW are close to those of the Cyclone base-case[53], $\rho_{*}=2-4 \times 10^{-3}, R / L_{T}=6.9, R / L_{n}=2.2$, $\epsilon=0.19$. ORB5 simulations have been run without source terms. To have a phase of slowly-decaying turbulence with quasi-stationary profiles[44], a smaller value of $\rho_{*}$ has been considered, typically $\rho_{*}=4 \times 10^{-4}$. The gradient length for the ion temperature during the decaying-turbulence phase stays about $R / L_{T} \approx 7$ in the region $0.5 \lesssim \sqrt{\psi} \lesssim 0.9$ (where $\sqrt{\psi}$ is used as a normalized radial coordinate and the resonant surface is located at $\sqrt{\psi}=0.67)$. The background density was assumed to be flat, $R / L_{n}=0$. A small inverse aspect ratio, $\epsilon=0.14$, has been considered. The whole radial domain is simulated. In the PIC simulations presented here, the new electromagnetic version of ORB5[54] has been used in the electrostatic approximation. With respect to previous PIC results[36], new code diagnostics have been implemented (e. g. on the local energy spectrum, see below), but the physical picture remains the same. In the following, it is shown that the findings of global, slowly-decaying turbulence simulations agree well with local flux-tube simulations as long as the physical model remains the same, in particular with respect to the treatment of 
the electron dynamics. As a matter of fact, both codes assume, for the case of an adiabatic electron response, that the electrons react instantaneously to the electrostatic potential on the unperturbed flux surfaces. In the presence of an island, however, the axisymmetry typical of the tokamak configuration is destroyed. The only feasible way to include the perturbed magnetic geometry in the electron response is to treat also the electrons as a kinetic species. At the moment, however, this is not possible with ORB5 if a magnetic island is present (for an unperturbed magnetic equilibrium with nested magnetic surfaces, ORB5 simulations of electromagnetic turbulence with kinetic electrons have instead been presented recently[54]). For this reason, modes with $m=n=0$ are excluded from ORB5 runs[36]. In this section, simulations that treat the electrons as a kinetic species are performed with the code GKW, employing the actual mass ratio of a deuterium plasma.

The first effect connected with the presence of the island seen in the simulations is the expected flattening of density and temperature profiles inside the island separatrix, after a transient phase in which they relax to the perturbed magnetic configuration. Just outside the separatrix, a steepening with respect to the unperturbed profiles is found, in particular in the region around $\xi=0$ (i. e. at the level of the O-point), where the perturbed flux surfaces are "compressed" by the island. However, a corresponding increase of the turbulence level is not observed. On the contrary, the heat flux and the heat conductivity are reduced in this region, while they are higher across the X-point, as shown in Fig. 2, where the eddies can cross the rational surface. In both codes, an asymmetry in the heat conductivity at the $\mathrm{X}$-point is usually seen, that can be attributed to the fact that the eddies are able to convect through the island in the drift direction. This convection mechanism seems to act more effectively than diffusive turbulence spreading[55] in transporting the turbulence towards linearly stable regions inside the island. The turbulence reduction at the separatrix for $\xi=0$ mentioned above is explained as a result of the action of the sheared flows connected with the long-wavelength components of the electrostatic potential, which "rip apart" the eddies in analogy to what is know from zonal-flows dynamics. Fig. 3 reports the time evolution of the local turbulence spectrum at two different radial locations in Fig. 2, namely $\langle\sqrt{\psi}\rangle=0.57$ and $\langle\sqrt{\psi}\rangle=0.63$ (angular brackets denoting the average value of the radial coordinate on the perturbed flux surface). Well outside the island, it can be seen that the $n=2$ component of the electrostatic potential, that decays during the first phase of the ORB5 simulation (in which the profiles adjust to the perturbed equilibrium[36]), is again 
pumped through a nonlinear coupling with the small-scale (turbulent) modes. However, the energy of the long-scale mode is smaller than that of turbulence during most of the simulation time and the turbulent eddies efficiently transport heat across the flux surfaces. On the contrary, at the island separatrix, especially on the side towards the plasma core, the $n=2$ mode largely dominates the other modes. The result observed in several simulations is a reduction of the heat flux at the separatrix. From the simulations performed so far[35], however, no firm conclusion can be drawn on whether non-linearly generated sheared flows arising in the presence of small magnetic islands can contribute to the improvement of the confinement in the vicinity of rational surfaces, which has been reported in experiments[56]. Inside the island, the $\mathbf{E} \times \mathbf{B}$ transport can be dominated by long-wavelength modes. The transport due to different modes is shown in Fig. 4.

The picture outlined above emerges consistently from ORB5 and GKW simulations with adiabatic electrons. The inclusion of the perturbed magnetic equilibrium in the electron response through kinetic electrons in GKW, however, shows that the dynamics of the longwavelength mode in the island region can change significantly. Fig. 5 shows a snapshot of the electrostatic potential, perturbed density, total density and total density profile across the $\mathrm{O}$ and X-point. A large-scale electrostatic mode, which we will call "vortex mode" following Ref. 17, develops in the centre of the magnetic island as a consequence of the non-linear flow of energy into long wavelengths discussed above. The time evolution of this structure exhibits a complex dynamics, being non-stationary with a periodic sign reversal of the electrostatic potential. In passing, we note that a nonzero zonal-flow frequency has been observed recently in turbulence simulations including a magnetic island in slab geometry[37]. It is shown in Ref. 35 that the heat flux associated with the vortex mode at its peak intensity can largely exceed the flutter flux, i. e. the radial flux due to the parallel motion of the particles along the perturbed magnetic-field lines. On average, the flutter flux and the $\mathbf{E} \times \mathbf{B}$ flux due to the vortex mode yield a comparable contribution to the total heat transport. The strength of the mode is found to be reduced for smaller $\rho_{*}$, i. e. larger scale separation between island and turbulence. This fact could also be related, however, to the different degree of flattening of the ion temperature inside the island between both cases and the associated different behaviour of the microinstabilities. The vortex mode appears also in simulations performed for the same plasma parameters in the case of islands rotating at the ion or electron diamagnetic frequency. 
IV. TURBULENCE-FREE GYROKINETIC SIMULATIONS OF ROTATING ISLANDS

We now turn to simulations performed with the code GKW, in which a filter is applied on high angular component of the spectrum of the electrostatic disturbances to suppress the turbulence. Moreover, the background density and temperature gradients are reduced, $R / L_{n} \leq 1$ and $R / L_{T} \leq 3$. Although neoclassical physics is still not fully tested in the code (and therefore collisions are switched off), the presence of fully kinetic electrons allows the investigation of the behaviour of the potential connected to the rotation of the island, and provides useful information on the density and the temperature in the presence of toroidal effects, which can be compared with kinetic simulations that retain neoclassical physics but not a self-consistent determination of the electrostatic potential[31]. It is worth to stress that numerical simulations can explore a parameter range which goes beyond the validity of the neoclassical analytic theory, in particular magnetic islands whose width is comparable or smaller than the ion banana width. As in the previous section, the island evolution is not computed and the (constant) island rotation frequency is treated as an input parameter.

A known result of tearing mode theory is that the potential associated to the rotation of a neoclassical magnetic island is $[7,8]$

$$
\tilde{\phi}=\frac{\omega q}{m c}\left[\psi-\psi_{s}-h(\Omega)\right]
$$

as can be derived from requiring $E_{\|}=-\nabla_{\|} \tilde{\phi}-(1 / c) \partial \tilde{A}_{\|} / \partial t=0$. The function $h(\Omega)$ is an integration "constant" (deriving from the fact that $\nabla_{\|} \Omega=0$, see Section II) which is connected with the density profile in the presence of an island[8]. Assuming no sources nor sinks in the island and a vanishing electric field away from it, $h(\Omega)$ must vanish inside the island separatrix for parity reasons (this corresponds to profile flattening), and must scale as $\psi-\psi_{s}$ for $\psi-\psi_{s} \gg W_{\psi}$. The $\mathbf{E} \times \mathbf{B}$ velocity connected with this electrostatic potential forces the plasma inside the island to co-rotate with it, while it vanishes at some distance from the island, where $\tilde{\phi}$ attains a constant value. When a finite island rotation is enforced in GKW simulations, and the island width is larger than the ion orbit width, a potential with the shape given by Eq.(6) develops, Fig. 6a. Its peak value (at the island separatrix) exhibits the expected linear scalling with the rotation frequency $\omega$, while it vanishes at the edge of computational domain to satisfy the periodic boundary conditions. If the rotation frequency 
is chosen to be in the ion diamagnetic direction, $\tilde{\phi}$ is found to be localized in the vicinity of the island, while its shape is broader if the rotation is in the electron direction. It is known that islands rotating at a frequency between 0 and the electron diamagnetic frequency lead to the emission of drift waves[23]. In this case, the maximum of the electrostatic potential outside the island is found to be localized between the $\mathrm{O}$ and the $\mathrm{X}$-point (Fig. 6b), as found in analytic theory for the slab case[25].

A set of simulations has been performed at small island width (slightly smaller than the thermal ion banana width) in order to determine the density and temperature profiles in a rotating island. In recent drift-kinetic simulations[31], it was found that, when the ratio $\rho_{i} / W$ is increased, the ion density perturbation exhibits more and more an adiabatic ("unmagnetized") response[22, 57]. As a consequence, due to the shape of the electrostatic potential, cf. Eq. (6), the ion density perturbation was observed to lead to a flattening of the total density profile even in small islands rotating in the ion diamagnetic direction, and to a steepening for islands rotating in the opposite direction. Correspondingly, the bootstrap current was found to be strongly reduced in islands rotating at the ion diamagnetic frequency and largely preserved when the island frequency equals the electron diamagnetic frequency, implying an enhanced neoclassical stability in the latter case. Since, however, in the presence of an unflattened ion density quasi-neutrality was violated (the electron orbit width is always much smaller than the island width and the electron density tends therefore to be flat), in Ref. 31 the (prescribed) electrostatic potential was modified with respect to Eq. (6) in order to recover quasi neutrality. In the simulations presented here, the potential is determined self-consistently form the electron response. GKW simulations show that the ion density profile exhibits the same properties as described above, the electron density profile following closely that of the ions. As can be clearly observed in Fig. 7, for rotation frequencies above the diamagnetic frequency, the density perturbation leads even to a positive density gradient inside the island (rotation in the ion direction) or to a gradient larger than the equilibrium one (rotation in the electron direction). In other words, these simulations support the neoclassical drift kinetic simulations of Bergmann at al. [31], according to which the neoclassical drive is largely suppressed in a small island rotating in the electron diamagnetic direction. 


\section{SELF-CONSISTENT GYROFLUID SIMULATION OF THE ISLAND EVOLU- TION IN THE PRESENCE OF TURBULENCE}

The self consistent interaction between magnetic islands and turbulence was studied by the GEMZ model described above. The nonlinear dynamics is followed together with local modifications in the profiles but the MHD and neoclassical flow equilibrium is removed by separating $n_{z} \rightarrow n_{0}+\tilde{n}_{z}$ (e.g., for the gyrocentre density of species $z$ ) for each dependent variable. Profile functions $n_{0}$ and $T_{0}$, set equal for ions and electrons, provide gradient dynamics for the turbulence. Tearing and island dynamics are enabled by keeping $u_{e 0}=-J_{0} / n e$ in the parallel velocity for the electrons, consistent with the chosen profile $q(x)$. The equilibrium dynamics[58] is avoided by leaving these profile functions out of the curvature terms. A small island, added consistently to $\tilde{A}_{\|}$and $\tilde{J}_{\|}$as a piece proportional to a simple radial profile and to $\cos \xi_{0}=\cos (2 \theta-\varphi)$ (here, a 2,1 island is considered), is combined with the random-bath turbulent density fluctuations in the initial state.

The plasma parameters are typical of tokamak core conditions $\left(n=3 \times 10^{19} \mathrm{~m}^{-3}\right.$ and $T=2 \mathrm{keV}$ and $B_{0}=2.5 \mathrm{~T}$ ) in the dimensions $R_{0}=1.65 \mathrm{~m}$ and $a=0.5 \mathrm{~m}$. The profiles have $R / L_{T}=8$ and $L_{n} / L_{T}=3$. Various current profiles were tried. An "exponential" case has $q=4 \exp \left(r_{a}^{2}-1\right)$, a parabolic case has $q=1+3 r_{a}^{2}$ and a tearing unstable case $q=3 /\left(2-r_{a}^{2}\right)$ taken from Wesson [59], where $r_{a}=\exp \left(x_{c}\right)$ is the conformal normalized minor radius. In each case $J_{0}=\left(B_{0} / \mu_{0} R_{0}\right)\left(1 / r_{a}^{2}\right)\left(\partial / \partial r_{a}\right)\left(r_{a}^{2} / q\right)$ is chosen consistently. The poloidal and toroidal flux profiles $\psi$ and $\psi_{t}$ do not enter the equations but are used in the helical flux diagnostic $\Psi=R_{0}^{-1}\left[\left(\psi_{t} / 2-\psi\right)-\operatorname{Min}\left(\psi_{t} / 2-\psi\right)\right]+\tilde{A}_{\|}$of the $q=2$ rational surface (the flux label $\Omega$ introduced in Section II can be easily derived from $\Psi$ through a second-order expansion of $\psi_{t}$ around the rational surface). Here, the sign convention is for $\mathbf{B}=I \nabla \varphi+\nabla \varphi \times \nabla \psi$ and $\nabla R \times \nabla Z \cdot \nabla \varphi>0$.

The current profiles in present tokamaks are usually mildly stable to tearing for the cylindrical model and neoclassical effects are usually invoked to account for observed magnetic fluctuations consistent with magnetic islands a few $\mathrm{cm}$ wide. It is important to note that the combination of gradients and toroidal curvature are severely stabilizing for tearing modes[10], due to the action of geodesic curvature (the part acting on $\partial / \partial \psi$ of the disturbance). Hence the maintenance of the island has to be a robust effect. Turbulence has two potential effects on islands. Diffusion is more familiar, but the details of drift wave 
turbulence[60, 61] show that this has to act through the pressure and current fluctuations, not the $\mathbf{E} \times \mathbf{B}$ or magnetic part. The other potential effect is inverse cascade dynamics through these two components. For drift waves dynamically incompressible vorticity dynamics is active. In addition for electromagnetic cases one has the inverse helicity cascade in 2D MHD turbulence[62], which directly affects $\widetilde{A}_{\|}$. Normally the nonlinear electromagnetic processes are weaker than the $\mathbf{E} \times \mathbf{B}$ ones[63]. However, in this case they have the island's component of $\tilde{A}_{\|}$to act upon and are therefore non-negligible.

A further less-familiar component of this dynamics from the point of view of magnetic island studies is that the turbulence is dominantly ion temperature gradient (ITG) driven despite the presence of many other mode types and despite the mild finite-beta stabilization[50]. The tendency of the electron dynamics to equalize the pressure along field lines and within the island does not affect ion temperature fluctuations, so the effect to weaken the turbulence is negligible.

The main result of the computations so far is that the initial long-wave disturbance in $\tilde{A}_{\|}$is not only maintained but increased during the turbulence which is well saturated. The simulations show an island activity which survives in the face of the curvature/toroidicity effects. The helical flux diagnostic $\Psi$ forms a channel whose width depends on the magnitude of $\tilde{A}_{\|}$: the contour interval is $-1 / 3$ times the minimum value of $\Psi$, so the channel is wide for larger islands. The visual appearance is therefore indicative of island robustness. The form of a 2/1 island indicates island coherence and is confirmed by a corresponding peak in the disturbance spectrum, as presented in Fig. 8. A coupling to the $m=3$ mode is also visible. The strength of this coupling also fluctuates as the island evolves. The tearing-unstable case shown in the figure exhibits a more clear and more coherent island but the other two cases also show strong activity albeit with less island coherence. The island activity persists despite the absence of a clear unstable-eigenmode signature in $\tilde{A}_{\|}$- one looks for a slope discontinuity in the O-point cut of $A_{\|}$across the island, which shows neither an obvious eigenmode structure nor significant chopping by turbulence. Indeed the poloidal spectrum of $A_{\|}$shows clear dominance by the $m=2$ component. The control cases without turbulence but with all the toroidal and pressure gradient effects are yet to be run; these and more detailed energy transfer diagnosis will provide for more definitive findings which will be published in the future. 


\section{SUMMARY AND CONCLUSIONS}

In this paper, recent advances in numerical simulations of tearing modes in toroidal geometry, including FLR effects have been reported. The development of highly parallelized and thoroughly benchmarked gyrokinetic and gyrofluid codes, as well as the increasing availability of high-performance computer resources allow the first steps in this area, employing realistic plasma parameters. Three different approaches have been followed here. The first one relies on the fact that the tearing mode grows on a much longer time scale than the turbulence. The magnetic island can then be implemented as a static magnetic perturbation and the development of the microinstabilities retaining toroidicity and kinetic effects can be investigated. Our results for electrostatic ITG turbulence confirm previous findings[36] that outside the island the fluctuations are much larger at the $\mathrm{X}$-point as compared to the the O-point, where sheared flows associated to nonlinearly-driven long-wavelength modes suppress the turbulence even in the presence of increased gradients. Inside the island, the turbulence is reduced because of the flattening of temperature and density profiles due to the fast parallel motion along the perturbed field lines. Eddies convected in the drift direction contribute significantly to the turbulence level inside the island in the region close to the X-point. The nonlinear coupling between small (turbulence) and large (island) scales can generate a vortex mode that can yield inside the island a transport level comparable to the parallel (flutter) transport. This mode is found only if the perturbed magnetic geometry is accounted for in the electron response. These results show that the transport in the island region has more complex behaviour than in the "standard" paradigm[20] and confirm the importance of the coupling between small and large scale. From a computational point of view, moreover, they represent a cross-check that different approaches (local and global) to the solution of the gyrokinetic equation in the presence of an island yield a consistent physical picture if the same assumptions are made. The second method presented here again employs a toroidal gyrokinetic formalism, but excludes the turbulence. The long-term goal is in this case to study processes taking place on the collisional time scale. Although the collision operator is still not fully functional, first collisionless results for an island with an imposed rotation reveal the potentialities of this approach, which allows a determination of the electrostatic potential associated to the island rotation, of the density and current profiles. Finally, the gyrofluid simulations performed with the GEMZ code demonstrate -for 
the first time for realistic tokamak parameters - the role of electromagnetic turbulence for the growth of the tearing mode, whose evolution is calculated in this case self-consistently as a part of the global disturbance spectrum.

\section{ACKNOWLEDGMENTS}

The simulations presented in this paper have been partly performed on the HPC-FF machine at the Jülich Supercomputing Centre, Germany.

[1] H. P. Furth, J. Killeen, and M. N. Rosenbluth, Physics of Fluids 6, 459 (1963).

[2] P. H. Rutherford, Physics of Fluids 16, 1903 (1973).

[3] O. Sauter et al., Physics of Plasmas 4, 1654 (1997).

[4] W. X. Qu and C. J. D, University of Wisconsin Plasma Report UWPR 85-5 (1985).

[5] R. Carrera, R. D. Hazeltine, and M. Kotschenreuther, Physics of Fluids 29, 899 (1986).

[6] Z. Chang et al., Physical Review Letters 74, 4663 (1995).

[7] A. I. Smolyakov et al., Physics of Plasmas 2, 1581 (1995).

[8] H. R. Wilson et al., Physics of Plasmas 3, 248 (1996).

[9] A. H. Glasser, J. M. Greene, and J. L. Johnson, Physics of Fluids 18, 875 (1975).

[10] M. Kotschenreuther, R. D. Hazeltine, and P. J. Morrison, Physics of Fluids 28, 294 (1985).

[11] C. C. Hegna, Physics of Plasmas 6, 3980 (1999).

[12] M. Yamada, R. Kulsrud, and H. Ji, Reviews of Modern Physics 82, 603 (2010).

[13] F. L. Waelbroeck, Nuclear Fusion 49, 104025 (2009).

[14] P. Kaw, E. Valeo, and P. Rutherford, Physical Review Letters 43, 1398 (1979).

[15] K. Itoh, S.-I. Itoh, and A. Fukuyama, Physical Review Letters 69, 1050 (1992).

[16] P. H. Diamond, R. D. Hazeltine, Z. G. An, B. A. Carreras, and H. R. Hicks, Physics of Fluids 27, 1449 (1984).

[17] C. J. McDevitt and P. H. Diamond, Physics of Plasmas 13, 032302 (2006).

[18] A. Sen, R. Singh, D. Chandra, P. Kaw, and D. Raju, Nuclear Fusion 49, 115012 (2009).

[19] S.-I. Itoh, K. Itoh, and M. Yagi, Plasma Physics and Controlled Fusion 46, 123 (2004).

[20] R. Fitzpatrick, Physics of Plasmas 2, 825 (1995).

[21] F. Militello, F. L. Waelbroeck, R. Fitzpatrick, and W. Horton, Physics of Plasmas 15, 050701 
(2008).

[22] J. W. Connor and H. R. Wilson, Physics of Plasmas 2, 4575 (1995).

[23] F. L. Waelbroeck, J. W. Connor, and H. R. Wilson, Physical Review Letters 87, 215003 (2001).

[24] M. James and H. R. Wilson, Plasma Physics and Controlled Fusion 48, 1647 (2006).

[25] H. R. Wilson and J. W. Connor, Plasma Physics and Controlled Fusion 51, 115007 (2009).

[26] R. D. Sydora, Physics of Plasmas 8, 1929 (2001).

[27] W. Wan, Y. Chen, and S. E. Parker, Physics of Plasmas 12, 012311 (2005).

[28] F. L. Waelbroeck, F. Militello, R. Fitzpatrick, and W. Horton, Plasma Physics and Controlled Fusion 51, 015015 (2009).

[29] E. Poli, A. G. Peeters, A. Bergmann, et al., Physical Review Letters 88, 075001 (2002).

[30] E. Poli et al., Plasma Physics and Controlled Fusion 45, 71 (2003).

[31] A. Bergmann, E. Poli, and A. G. Peeters, Physics of Plasmas 16, 092507 (2009).

[32] E. Poli, A. Bergmann, and A. G. Peeters, Physical Review Letters 94, 205001 (2005).

[33] E. Poli, A. Bergmann, A. G. Peeters, L. Appel, and S. D. Pinches, Nuclear Fusion 45, 384 (2005).

[34] M. Siccinio and E. Poli, Plasma Physics and Controlled Fusion 51, 075005 (2009).

[35] W. A. Hornsby et al., Physics of Plasmas 17, 092301 (2010).

[36] E. Poli, A. Bottino, and A. G. Peeters, Nuclear Fusion 49, 075010 (2009).

[37] J. Li, Y. Kishimoto, Y. Kouduki, Z. X. Wang, and M. Janvier, Nuclear Fusion 49, 095007 (2009).

[38] A. Ishizawa and N. Nakajima, Nuclear Fusion 47, 1540 (2007).

[39] M. Muraglia, O. Agullo, M. Yagi, et al., Nuclear Fusion 49, 055016 (2009).

[40] S. Jolliet, A. Bottino, P. Angelino, et al., Computer Physics Communications 177, 409 (2007).

[41] A. Bottino, A. G. Peeters, R. Hatzky, et al., Physics of Plasmas 14, 010701 (2007).

[42] A. G. Peeters and D. Strintzi, Physics of Plasmas 11, 3748 (2004).

[43] A. G. Peeters, Y. Camenen, F. J. Casson, W. A. Hornsby, A. P. Snodin, D. Strintzi, and G. Szepesi, Computer Physics Communications 180, 2650 (2009).

[44] B. D. Scott, Physics of Plasmas 12, 102307 (2005).

[45] T. S. Hahm, Physics of Fluids 31, 2670 (1988).

[46] R. B. White et al., Physics of Fluids 26, 2958 (1983). 
[47] M. A. Beer, S. C. Cowley, and G. W. Hammett, Physics of Plasmas 2, 2687 (1995).

[48] A. G. Peeters, D. Strintzi, Y. Camenen, et al., Physics of Plasmas 16, 042310 (2009).

[49] B. D. Scott, Plasma Physics and Controlled Fusion 45, A385 (2003).

[50] B. D. Scott, Plasma Physics and Controlled Fusion 48, B277 (2006).

[51] T. Ribeiro and B. Scott, IEEE Trans. Plasma Sci. 38, 2159 (2010).

[52] B. D. Scott, Physics of Plasmas 8, 447 (2001).

[53] A. M. Dimits, G. Bateman, M. A. Beer, et al., Physics of Plasmas 7, 969 (2000).

[54] A. Bottino et al., IEEE Trans. Plasma Sci. 38, 2129 (2010).

[55] T. S. Hahm, P. H. Diamond, Z. Lin, et al., Plasma Physics and Controlled Fusion 46, A323 (2004).

[56] E. Joffrin, C. D. Challis, G. D. Conway, et al., Nuclear Fusion 43, 1167 (2003).

[57] S. V. Konovalov, A. B. Mikhailovskii, V. S. Tsypin, and S. E. Sharapov, Doklady Physics 47, $488(2002)$.

[58] B. D. Scott, Contributions to Plasma Physics 46, 714 (2006).

[59] J. Wesson, Tokamaks (Clarendon Press, Oxford, 1997).

[60] B. D. Scott, Physics of Fluids B 4, 2468 (1992).

[61] B. D. Scott, New Journal of Physics 4, 52 (2002).

[62] D. Biskamp, Nonlinear Magnetohydrodynamics (Cambridge University Press, Cambridge, 1993).

[63] S. Camargo, B. D. Scott, and D. Biskamp, Physics of Plasmas 3, 3912 (1996). 


\section{Figure captions}

Fig. 1: Implementation of a magnetic island satisfying periodicity conditions in the flux-tube code GKW: simple projection on radial Fourier harmonics, showing the deformation of the edge cells to ensure periodicity (a) and smoothing obtained by adding a Gaussian damping on higher harmonics (b).

Fig. 2: ORB5 simulation showing the heat flux in the island region with the associated the strong transport through the X-points $(\xi=\mp \pi)$ and its reduction on the island separatrix (marked by thick vertical lines) at the O-point location $(\xi=0)$.

Fig. 3: Time evolution of the toroidal energy spectrum (logarithm of the mode energy normalized to $M_{i} c_{s}^{2}$ ) of the potential disturbances for $\langle\sqrt{\psi}\rangle=0.57$ (outside the island, a) and for $\langle\sqrt{\psi}\rangle=0.63$ (island separatrix, core side, b). In the latter case, only low mode numbers (corresponding to the magnetic island) are significantly excited.

Fig. 4: Radial electrostatic heat flux (integrated over the simulation box) driven by each component of the spectrum for different values of the ratio $W / \rho_{i}$ (GKW simulations).

Fig. 5: Snapshot of a GKW simulation showing the electrostatic potential (top left), $\delta n / n$ (top right), the total density (bottom left) and the density profiles (bottom right; the green line represents the unperturbed profile, the blue line a cut through the O-point and the red line a cut through the $\mathrm{X}$-point).

Fig. 6: Electrostatic potential arising from a rotating magnetic island in the absence of turbulence for a frequency close to the electron diamagnetic frequency in the case of no equilibrium gradients (a) and with equilibrium gradients (b).

Fig. 7: Density profiles for a small island rotating in the ion diamagnetic direction (a) and in the electron diamagnetic direction (b).

Fig. 8: GEMZ simulations showing the contour plots of the helical flux $\Psi$ at two different time slices during the simulation, $t=20$ and $t=3150$ (time is expressed in $a / c_{s}$ units), and the disturbances spectrum (in terms of amplitude squared) for electron density (n), ion temperature (i), magnetic field (B), electrostatic potential (p) and parallel magnetic potential (A). 
Figures:
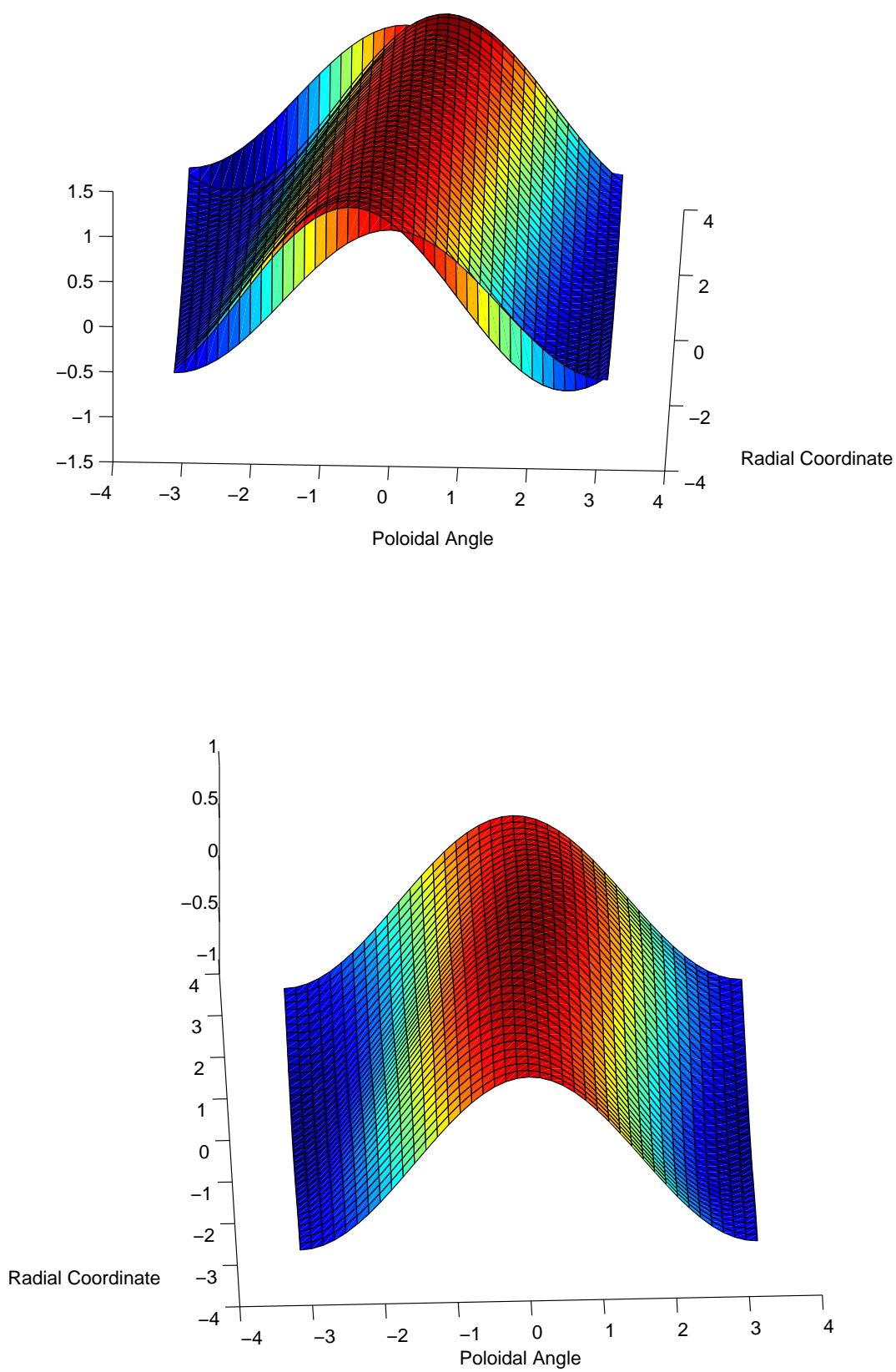

FIG. 1: 


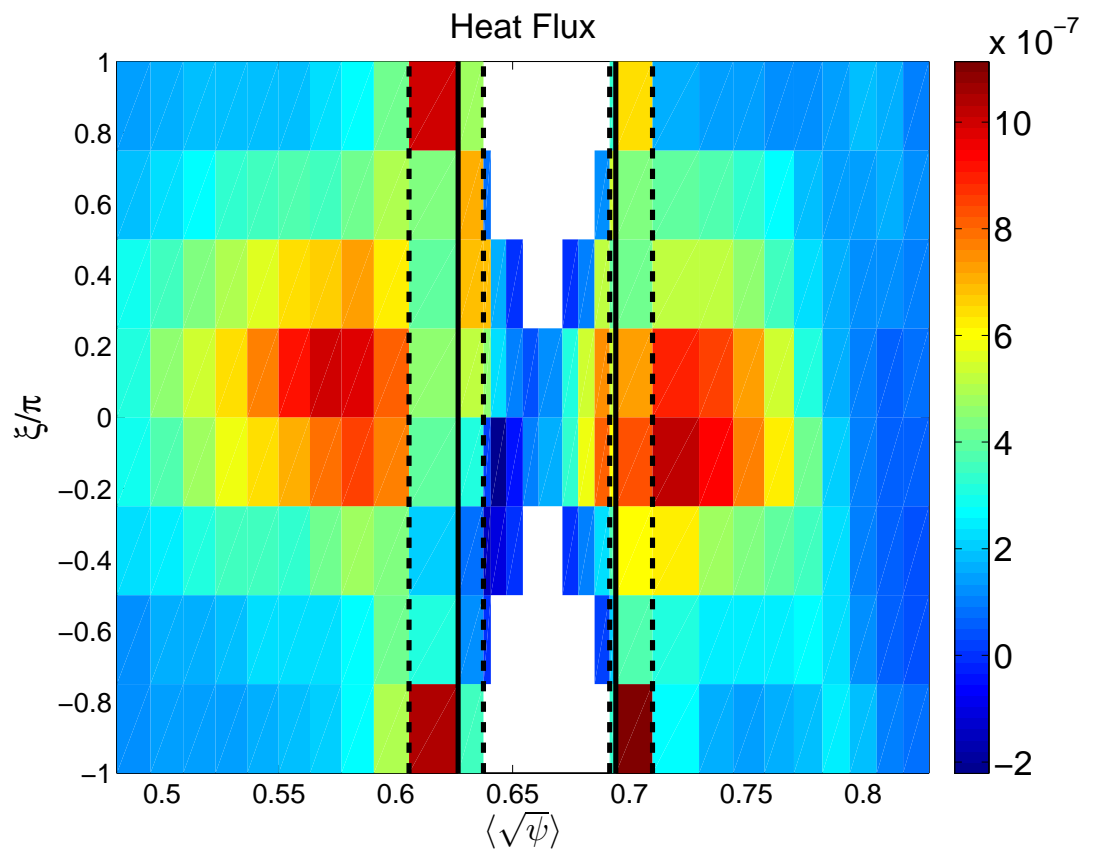

FIG. 2: 

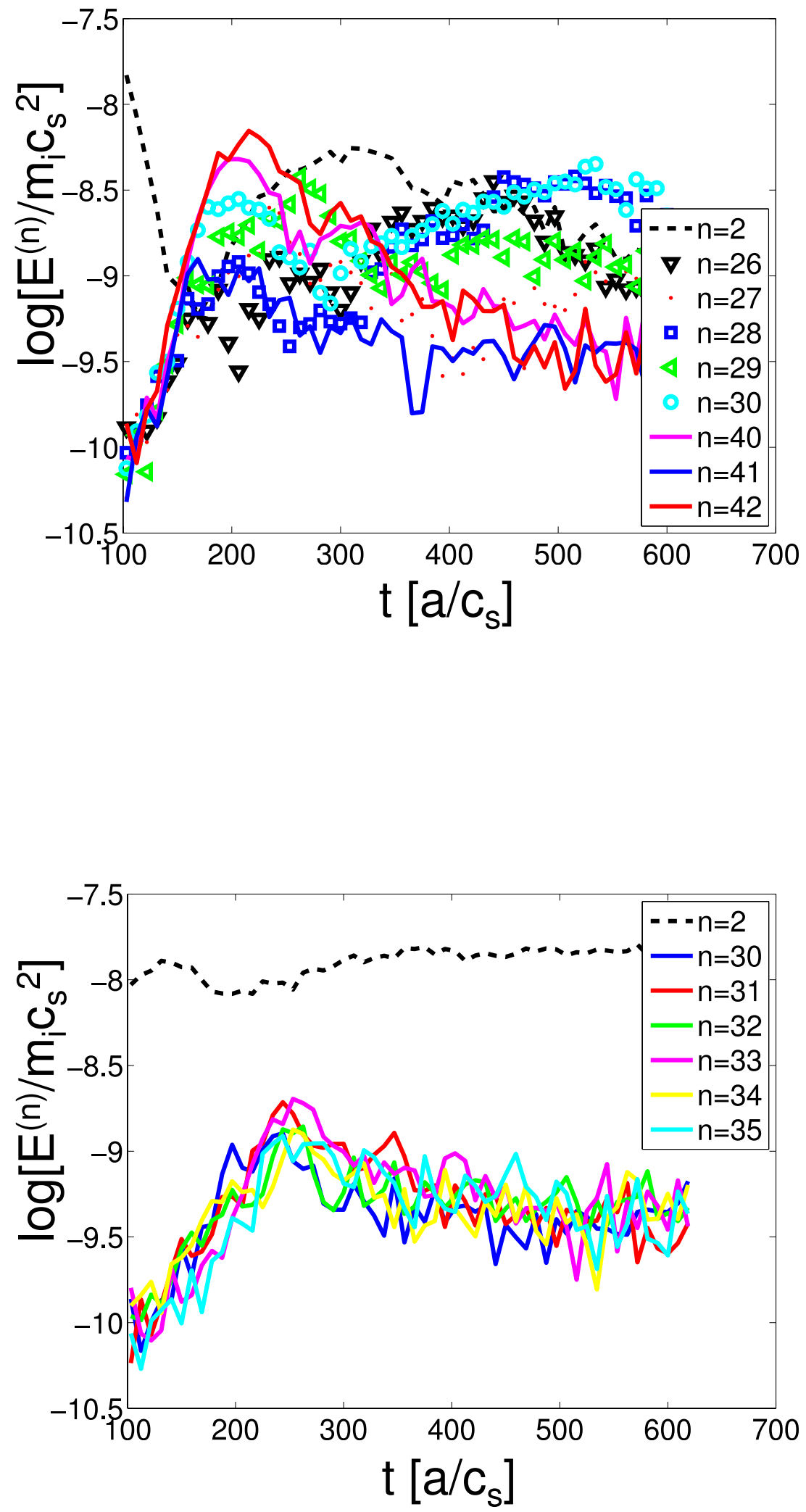

FIG. 3: 


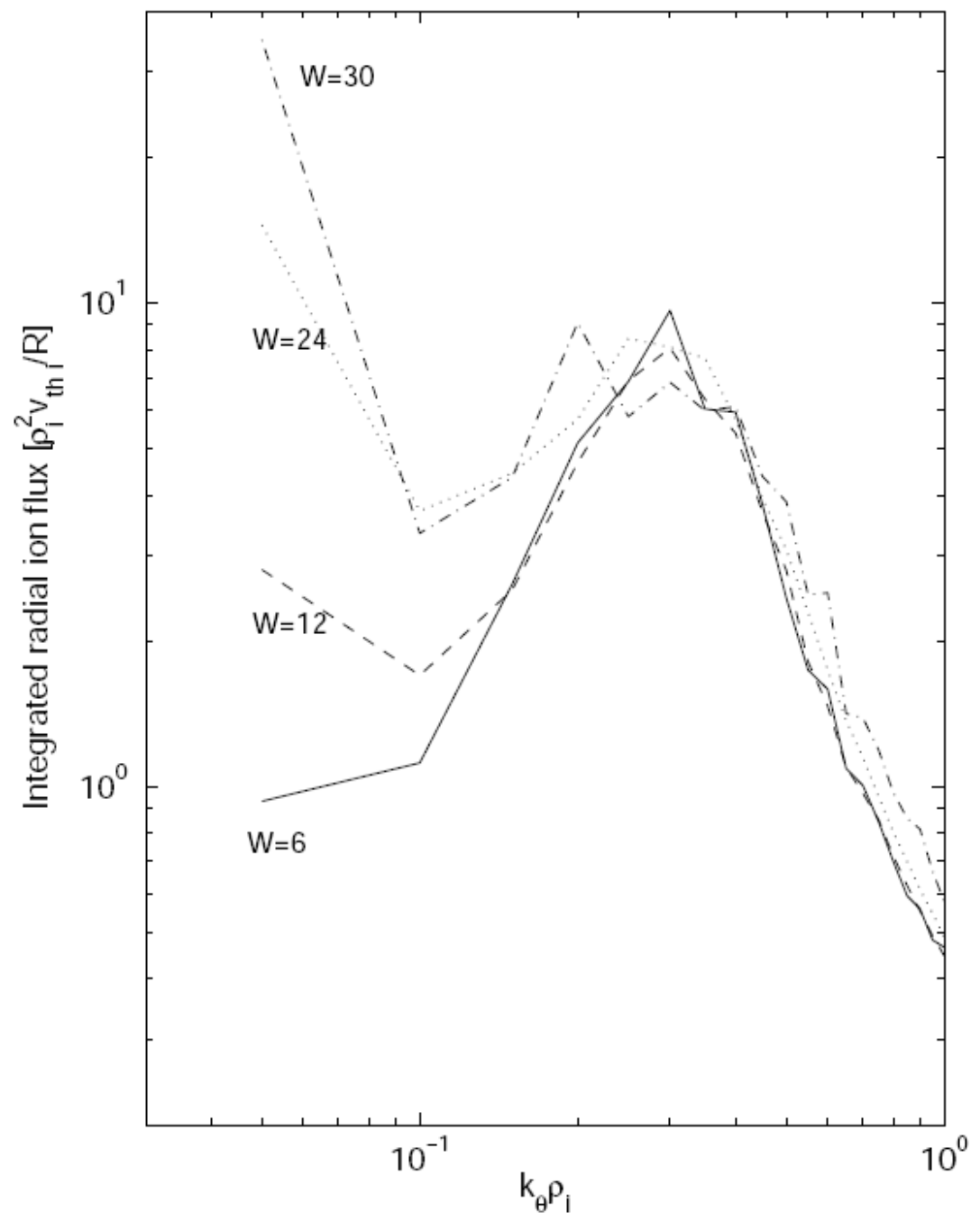

FIG. 4: 

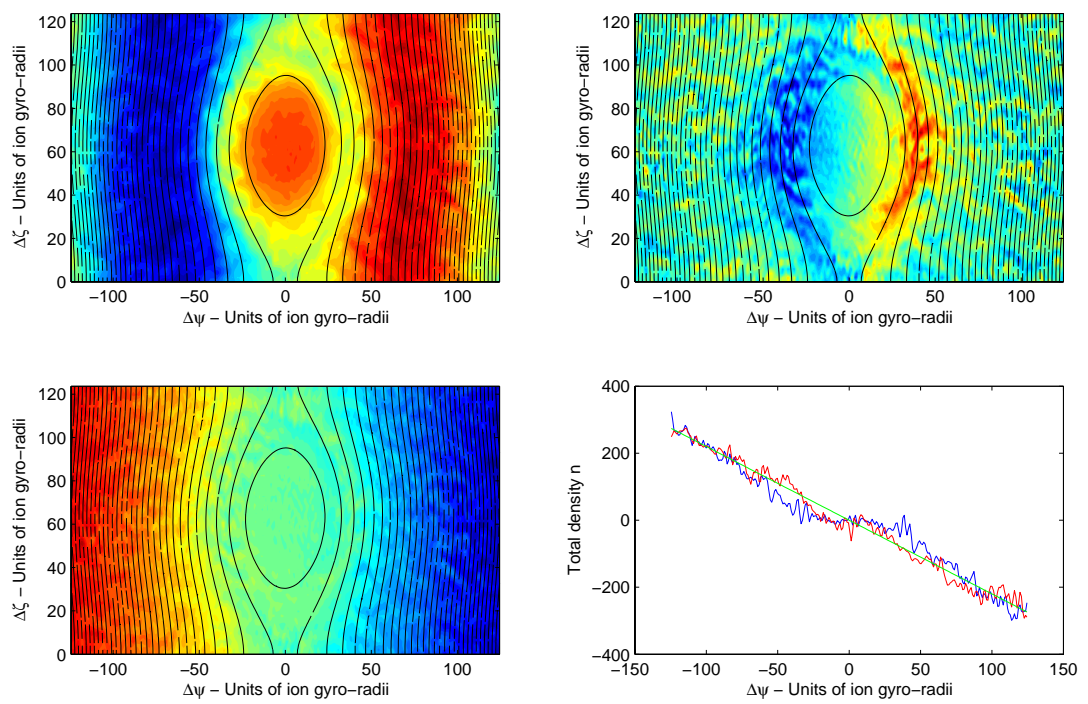

FIG. 5: 

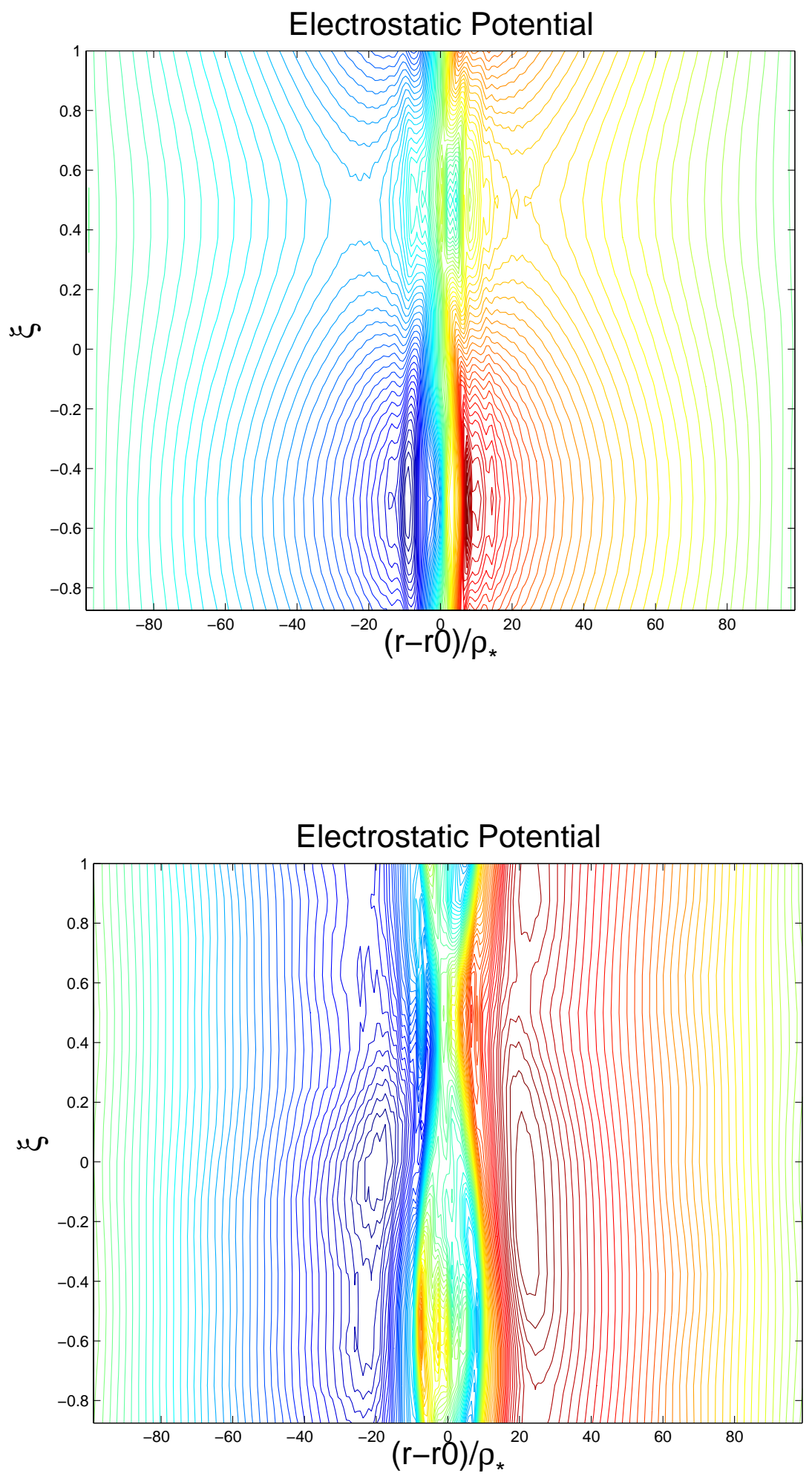

FIG. 6: 


\section{Total Density}

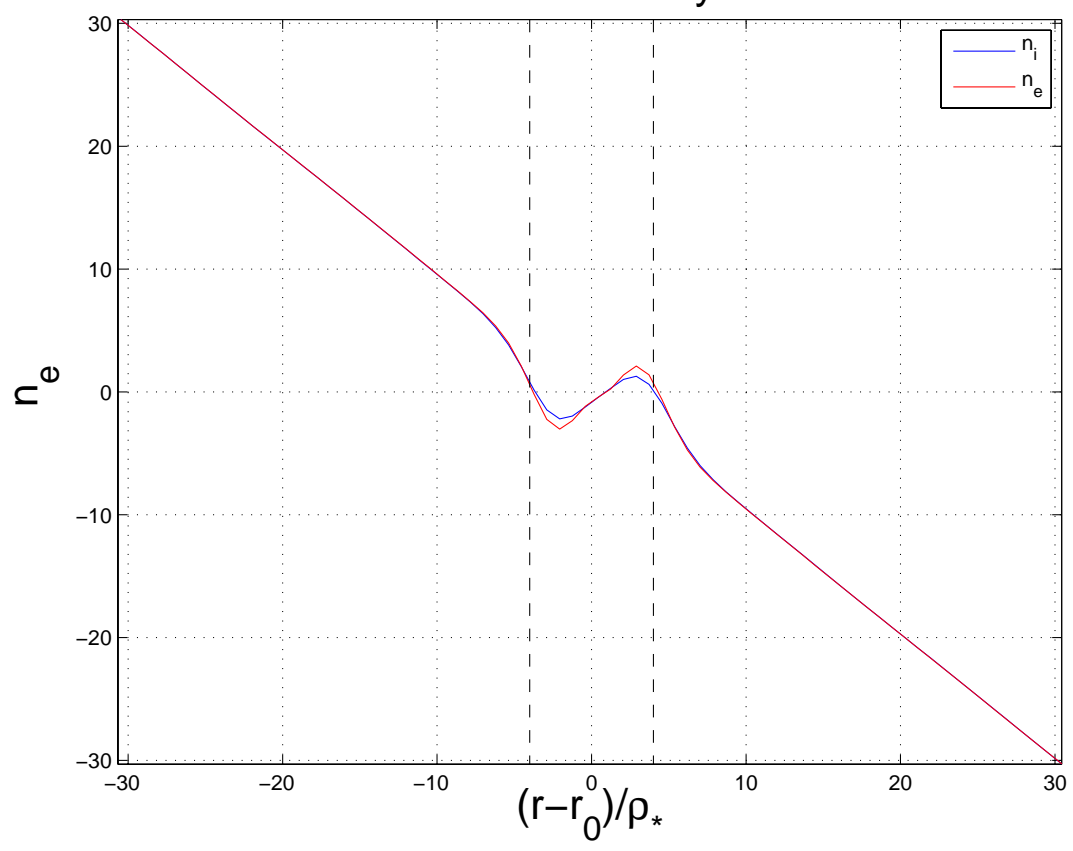

Total Density

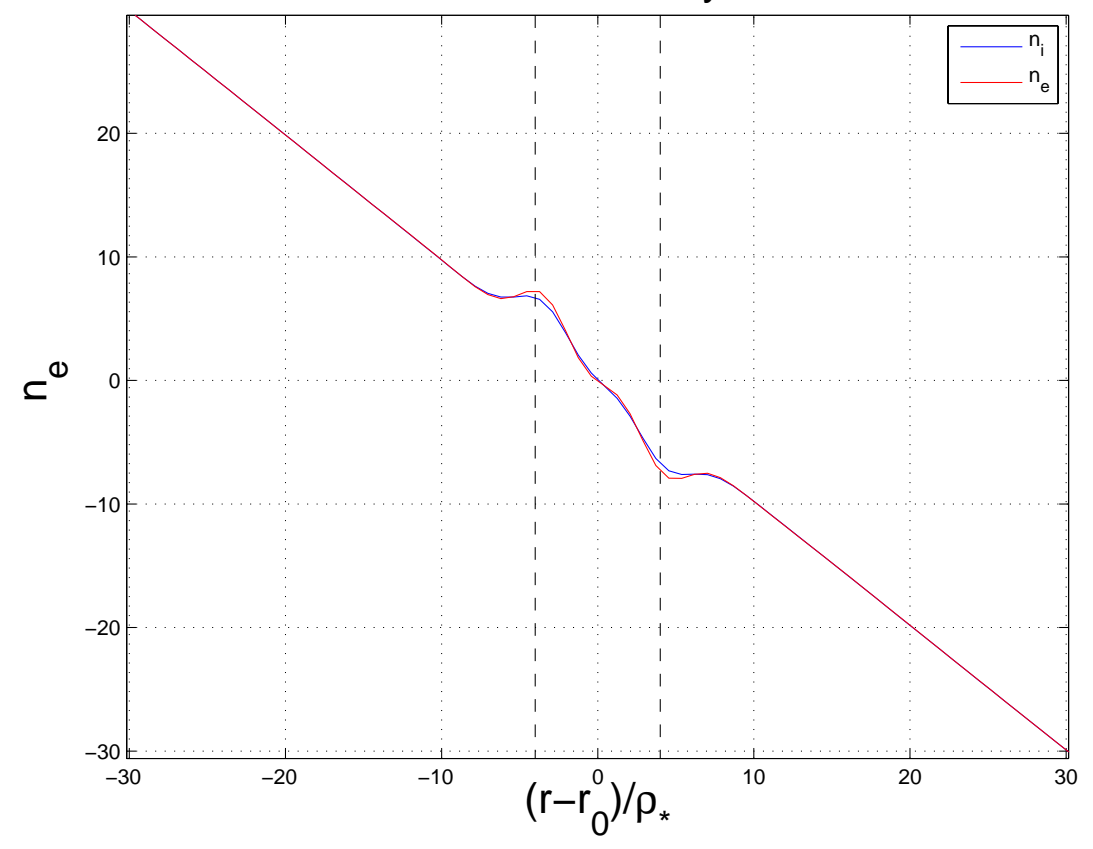

FIG. 7: 

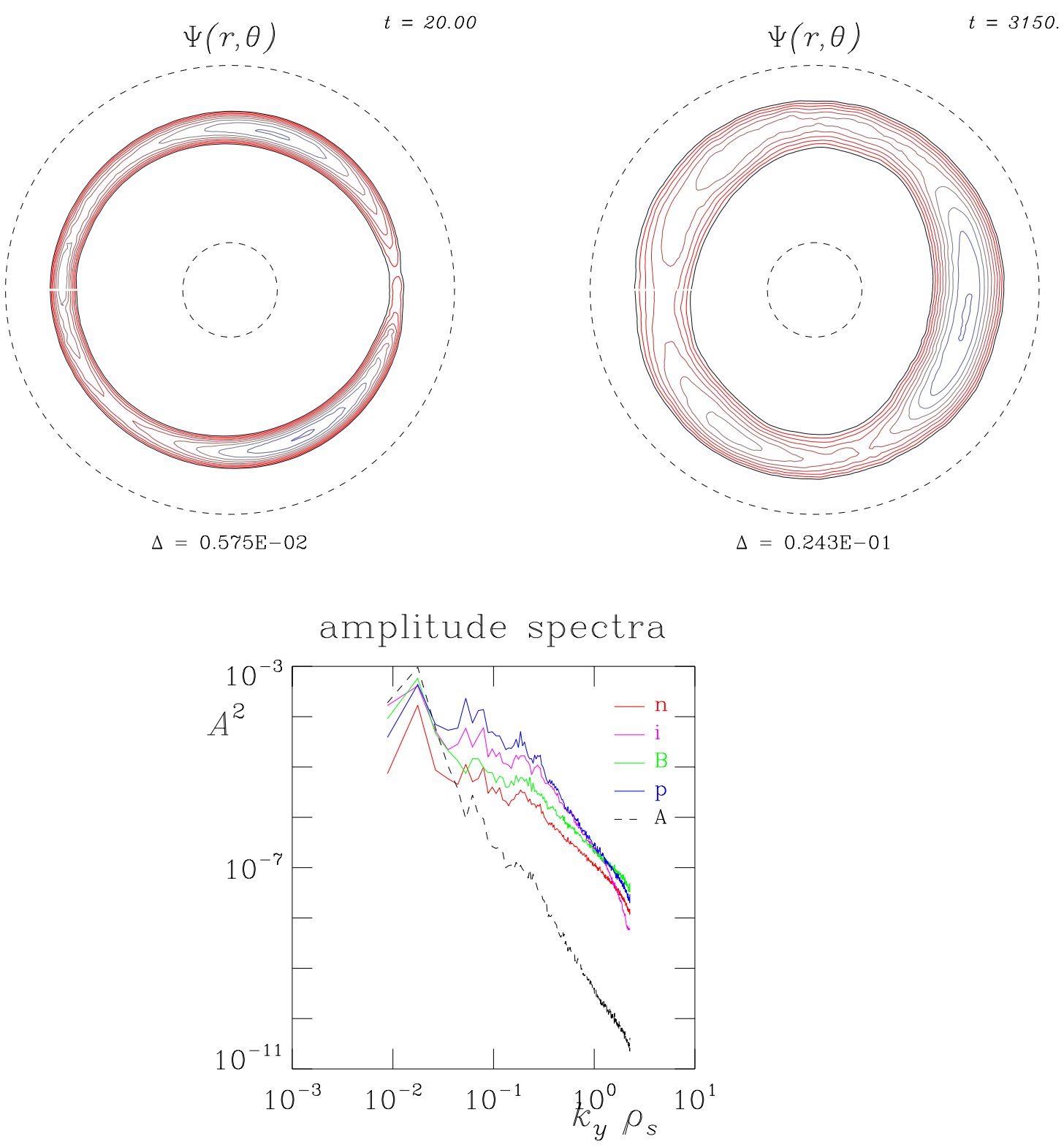

FIG. 8: 